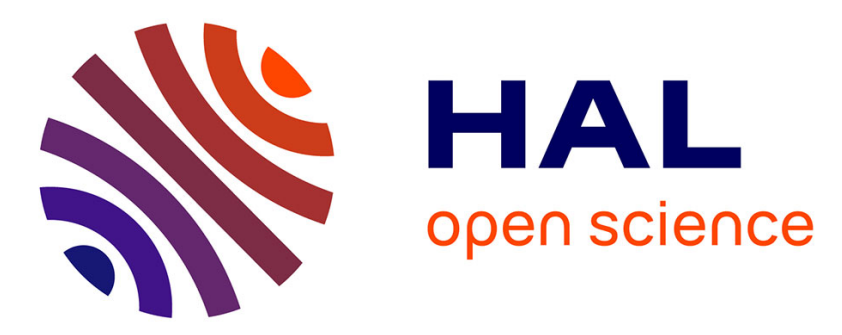

\title{
Les dynamiques d'usage des technologies de l'information et de la communication par les enseignants universitaires
}

Adel Ben Youssef, Walid Hadhri

\section{- To cite this version:}

Adel Ben Youssef, Walid Hadhri. Les dynamiques d'usage des technologies de l'information et de la communication par les enseignants universitaires. Réseaux: communication, technologie, société, 2009, 2009/3 (155), pp.23-54. 10.3917/res.155.0023 . halshs-00937210

\section{HAL Id: halshs-00937210 \\ https://shs.hal.science/halshs-00937210}

Submitted on 28 Jan 2014

HAL is a multi-disciplinary open access archive for the deposit and dissemination of scientific research documents, whether they are published or not. The documents may come from teaching and research institutions in France or abroad, or from public or private research centers.
L'archive ouverte pluridisciplinaire HAL, est destinée au dépôt et à la diffusion de documents scientifiques de niveau recherche, publiés ou non, émanant des établissements d'enseignement et de recherche français ou étrangers, des laboratoires publics ou privés. 
LES DYNAMIQUES D'USAGE DES TECHNOLOGIES DE L'INFORMATION ET DE LA COMMUNICATION PAR LES ENSEIGNANTS UNIVERSITAIRES : LE CAS DE LA FRANCE

Adel Ben YOUSSEF

Walid HADHRI 
Les technologies de l'Information et de la Communication (TIC) offrent de réelles potentialités pour accroître significativement la qualité de l'enseignement supérieur et pour modifier notre rapport au savoir (OCDE, 2005). Force est de reconnaître, qu'en dépit de deux décennies de politiques d'équipement intensif les changements des pratiques et des modèles pédagogiques demeurent faibles en Europe et plus particulièrement en France. En effet, le potentiel de ces technologies ne semble pas être totalement exploré "The Potential of ICT in Higher Education is not fully used". Dans une certaine mesure on pourrait parler d'un "paradoxe de la productivité» du secteur universitaire (Sharpe, 2004 ; Ben Youssef, 2005). Alors que l'équipement ne cesse d'augmenter, l'efficacité des dispositifs n'est pas totalement démontrée. La valeur pédagogique additionnelle est faiblement perçue. Cette situation semble d'autant plus problématique que la nouvelle génération d'étudiants est formée d'utilisateurs avancés de ces technologies (Digital Natives) ${ }^{1}$. L'écart entre leurs attentes en tant qu'utilisateurs de ces nouvelles technologies et l'offre proposée par les universités accentue la pression sur les enseignants du supérieur et met l'accent sur le rôle de ces derniers dans la mise en place des changements nécessaires ${ }^{2}$. De leurs attitudes, compétences, disponibilité et engagement pourraient dépendre les modifications structurelles attendues en matière de numérisation de l'enseignement supérieur'.

D'un point de vue analytique, une littérature pléthorique a cherché à comprendre les facteurs influençant l'adoption des TIC par les enseignants du supérieur ${ }^{4}$. Elle a permis de souligner une diversité de facteurs pouvant conduire les enseignants à adopter et intégrer les TIC dans leurs pratiques quotidiennes. On peut citer, sans exhaustivité, les facteurs contextuels, les facteurs sociaux, les facteurs personnels, les facteurs institutionnels et les facteurs pédagogiques.

Spécifiquement aux questions des usages et aux intensités d'usage, la littérature demeure faiblement développée. Deux raisons essentielles

\footnotetext{
${ }^{1}$ Rapport H. ISSAC, (2008)

${ }^{2}$ La première recommandation du Rapport ISSAC consiste à recommander l'offre de $100 \%$ des cours sous formats numériques pour $100 \%$ des étudiants.

${ }^{3}$ Cette étude a été conduite sous le programme de recherche eLene-EE (www.elene-ee.net). Elle a été financée par la Commission européenne - DG culture -

${ }^{4}$ Pour une synthèse de la littérature, le lecteur peut se rapporter à la thèse de C. RABY (Page 408).
} 
pourraient expliquer un tel constat. D'une part, la littérature, dans un premier stade, a confondu l'usage des technologies avec l'équipement. La disposition d'un ordinateur ou d'une connexion à l'Internet informe peu sur le temps consacré par les enseignants aux activités pédagogiques en ligne et aux modalités d'interactions avec leurs apprenants et leurs collègues. Ainsi, les études portant sur l'équipement et l'adoption avaient comme arrièrepensée d'aborder la question relative aux usages sans véritablement la traiter. D'autre part, la question relative aux usages et aux intensités d'usage constitue un axe de recherche naissant dans de nombreuses sciences sociales et il n'existe pas de méthodologie spécifique répondant à cette problématique. De réelles avancées récentes ont été effectuées dans les sciences économiques et de gestion et pourraient être transposées à l'étude des usages dans le milieu universitaire.

Partant de ces constats, cet article cherche à comprendre les déterminants d'usage des TIC par les enseignants du supérieur dans le milieu universitaire et à caractériser leur variété et leur intensité. Afin d'illustrer nos propos, nous avons administré une enquête auprès d'un échantillon de 615 enseignants universitaires en France durant l'année universitaire 2005/2006. Nous proposerons une démarche mesurant l'intensité d'usage des TIC dans le milieu universitaire permettant ainsi de qualifier les fractures numériques entre les divers groupes d'enseignants ${ }^{5}$.

Dans une première partie, nous proposons une revue de la littérature spécifique aux usages des TIC par les enseignants du supérieur. Dans une seconde partie, nous présenterons notre analyse descriptive des usages constatés et nous caractériserons les principales tendances observées. La troisième partie présentera un modèle économétrique permettant de relier les usages des enseignants en matière de TIC à leurs caractéristiques socioéconomiques ainsi que leur environnement du travail.

\section{UNE REVUE DE LA LITTERATURE PORTANT SUR LES USAGES DES TIC PAR LES ENSEIGNANTS DU SUPERIEUR}

De nombreuses études récentes cherchent à examiner de manière spécifique les usages par les enseignants universitaires des TIC. Au risque d'une simplification abusive, deux lignes de recherche sont identifiées. La

\footnotetext{
${ }^{5}$ Le questionnaire qui a été administré pendant le dernier trimestre 2005 et le premier trimestre 2006 en France à 615 enseignants dans 50 institutions universitaires françaises.
} 
première concerne la nature des usages en fonction des possibilités offertes par les nouvelles technologies. Il s'agit essentiellement d'identifier les modèles d'usage et de comprendre la nature des approches adoptées par les enseignants en fonction des contextes spécifiques d'apprentissage. La seconde ligne de recherche tend à montrer en quoi il existe des différences individuelles et collectives importantes en matière de compétences et de capacités cognitives entre les formateurs qui peuvent constituer des freins à un usage généralisé et optimisé de ces mêmes technologies. La thèse des fractures numériques peut être clairement défendue.

Spécifiquement à la première approche, de nombreuses études ont cherché à déterminer les modalités d'usage des TIC dans l'enseignement supérieur. Si dans un premier temps l'intérêt a été restreint à l'usage des technologies matures comme les ordinateurs et de l'Internet on constate de nos jours un engouement pour l'examen des usages relatifs aux nouvelles applications collaboratives issues du Web 2.0 (Blogs, Wikis, Plateformes...).

Basque (2005) montre que les TIC admettent diverses interprétations, en fonction de leurs usages réels dans l'expérience d'enseignement supérieur. De son point de vue, il convient de retenir trois interprétations. La première attribue le rôle de véhicule de message aux TIC. Ainsi, l'usage des TIC admet comme finalité de véhiculer les messages éducatifs. Un processus de substitution a lieu entre les médias traditionnels et les nouveaux médias. Dans la seconde interprétation, les TIC sont considérées comme un système symbolique au même titre que le langage. L'enseignement supérieur nécessite de ce point de vue d'adapter ses contenus au nouveau système symbolique. Enfin, dans une acception large, les TIC sont des outils cognitifs qui restructurent la pensée et les activités intellectuelles. Dans ce cadre les usages des TIC réorganisent de manière significative les activités d'apprentissage. Les TIC sont utilisées comme des artifacts cognitives. «Les ordinateurs sont généralement supposés pouvoir modifier la manière d'effectuer les tâches en amplifiant ou en élargissant nos capacités. L'hypothèse implicite est relative au fait que ces tâches demeurent les mêmes. Le point central que je souhaite souligner concerne le fait que le rôle premier des ordinateurs est de changer les tâches par une réorganization de notre fonctionnement mental, et non pas uniquement par le phénomène de l'amplification " (PEA, 1985, p. 168). Si l'on tient compte de cette grille de lecture, force est de reconnaitre que la troisième phase est faiblement constatée dans les études empiriques. 
Dans le contexte français, Albero et Dumont (2002), dans une étude de cas portant sur cinq universités françaises, montrent que l'usage des TIC s'insère davantage dans un schéma de substitution des canaux de délivrance des savoirs que dans un schéma de modification des pratiques de l'enseignement supérieur. Les nouveaux médias d'apprentissage semblent remplacer les anciens médias sans qu'un impact fort sur la nature de l'apprentissage soit constaté. Haeuw (2002) montre également dans une étude dans le contexte français que les représentations des apports des nouvelles technologies par les enseignants du supérieur en France restent pauvres. Le changement pédagogique nécessaire est faiblement exploré. L'usage n'obéit pas à un pattern particulier et les TIC sont considérés comme de simples outils compatibles avec un enseignement traditionnel. MEDLIN (2001) insiste sur la motivation des enseignants - les incitations intrinsèques et extrinsèques comme facteur principal déclanchant un usage efficace tourné avers une amélioration de la qualité de la formation. L'absence de motivation conduit à une faiblesse structurelle des usages en dépit d'une stabilisation des technologies et des applications.

Relativement aux nouvelles applications technologiques rendue possible par la nouvelle génération de l'Internet, de nombreuses études tendent à montrer un usage limité (Kessel, 2005, Underwood, 2005). Ici la thèse d'un retard de diffusion de ces technologies nous paraît davantage convenir.

Des constats similaires semblent être établis au niveau international. Au Canada et plus particulièrement au Québec, Lapierre et gingras (2001) montrent que les usages de l'Internet demeurent essentiellement orientés vers la recherche de l'information. Les impacts sur le processus d'apprentissage demeurent faibles. Karsenti (2005), à partir d'une enquête de terrain proche de la nôtre portant sur un échantillon de 709 enseignants du supérieur, révèle des usages relativement pauvres des possibilités offertes par les logiciels de présentation multimédias (comme PowerPoint). Basque (2005) montre que les usages des TIC au Canada par les enseignants du supérieur demeurent limités aux aspects informationnels et s'insère faiblement dans une optique plus large d'un changement profond de la nature de l'apprentissage. Coen (2006) met l'accent sur le fait que le défi actuel pour les enseignants consiste à transformer leur enseignement pour le rendre apte à capter les dividendes numériques. Il rejoint Larose et al. (2002, p.284) dans l'assertion selon laquelle « seules l'ampleur et la profondeur $d u$ changement de rapport au savoir ainsi qu'à la relation enseignementapprentissage peuvent être garantes de modifications significatives $d u$ 
rapport d'appropriation des outils didactiques par les enseignantes et enseignants ».

Relativement à la seconde approche, une littérature naissante a cherché à caractériser les différences d'usage des TIC selon les caractéristiques des enseignants. En effet, il s'agit ici de comprendre les impacts des caractéristiques socio-culturelles des enseignants sur l'intensité et la diversité des usages des TIC.

Becker, Ravitz et Wong (1999) ont montré qu'il existe des différences entre les enseignants du supérieur en matière d'expertise technique. Ces différences initiales influencent l'usage didactique des TIC chez les enseignants. Ainsi, les enseignants les plus experts adoptent davantage l'ordinateur et le font davantage utiliser par leurs étudiants. En outre, l'expertise influence le type d'utilisation des TIC et les compétences des étudiants. Les enseignants les plus experts en informatique sont davantage favorables aux utilisations les plus interactives. En revanche, les enseignants les moins experts se limitent souvent aux utilisations didactiques les plus faibles.

Andersson (2006) met l'accent sur l'effet de l'expérience sur les usages des TIC. Il a cherché à examiner en Suède la vulnérabilité des enseignants nouvellement nommés (New Qualified Teachers) dans les usages des TIC. Son étude tend à montrer des différences importantes entre les usages de cette population d'enseignants et le reste des enseignants. Il recommande une intégration d'un volet spécifique de formation aux enseignants nouvellement nommés.

D'autres études comme celle de Larose et al. (2002) ont cherché à montrer, à partir des travaux pionniers de Rogers (1995) en quoi la population des enseignants est partagée en cinq catégories dont les usages diffèrent substantiellement. Selon ces auteurs, les écarts dans les usages entre les enseignants proviennent essentiellement du degré d'expertise. Cette variable est fondamentale en matière d'impact sur les performances des étudiants : "l'exposition des étudiantes et des étudiants aux discours et aux pratiques d'utilisation des TIC par les enseignantes et les enseignants chevronnés et l'interaction avec ces derniers semblent constituer un des facteurs affectant les probabilités de transfert des compétences informatiques construites en milieu universitaire sur le plan des pratiques professionnelles des novices ».

Notre article cherche à concilier les deux lignes de recherche en proposant à la fois une méthodologie nouvelle développée en sciences économiques et de 
gestion pour décrire la variété et l'intensité des usages des TIC et l'applique au cas des enseignants du supérieur en France. Il propose également d'identifier les lignes de fractures dans l'intensité d'usage des TIC entre les diverses populations d'enseignants. Il permet ainsi de montrer des fractures qualitatives importantes.

\section{ANALYSE DESCRIPTIVE DE L'ADOPTION DES TIC PAR LES ENSEIGNANTS UNIVERSITAIRES}

Notre enquête a été réalisée auprès d'un échantillon aléatoire de 615 enseignants-chercheurs dans les universités françaises appartenant à une cinquantaine d'institutions universitaires. Notre échantillon est constitué de $52 \%$ enseignants de sexe masculin et de $48 \%$ de sexe féminin. Nous avons pris en compte la différence des statuts. Ainsi, $74 \%$ de l'échantillon est composé d'enseignants titulaires de leurs postes (Professeurs, Maître de Conférences, PRAG...) et $26 \%$ d'enseignants non-titulaires (vacataires et professionnels). La participation des enseignants à des dispositifs de formation par Internet constitue également un critère pouvant biaisé nos résultats. C'est pourquoi nous avons considéré $79 \%$ d'enseignants ne participant pas à des dispositifs de formation à distance via Internet et $21 \%$ d'enseignants participant en totalité ou en partie à des dispositifs à distance utilisant l'Internet. Ces proportions reflètent en grande partie le paysage de l'enseignement supérieur actuel.

En l'absence de statistiques relatives aux pratiques des enseignants en matière de TIC nous avons choisi d'opérer à l'aide d'une enquête par Internet. Ainsi, un biais important a été introduit et assumé dans cette étude. Notre enquête a été administrée seulement par courrier électronique en se basant sur les adresses électroniques des enseignants du supérieur. Cette technique nous a permis de faire deux rappels successifs et non pas un seul comme dans le cas des enquêtes par voie postale. Il est donc important de tenir compte du biais de sélection opéré et de la technique adoptée ${ }^{6}$.

\footnotetext{
${ }^{6}$ Le traitement des données a été automatisé afin de permettre une plus grande souplesse dans l'utilisation et dans le report de chaque réponse. Ainsi, nous avons élaboré un programme informatique, permettant d'enregistrer les réponses reçus par courriel dans un tableau Excel. Chaque réponse (observation) a été classée dans une ligne et chaque question en tant que variable. Ensuite, nous avons obtenu le tableau de manière à le traiter par des techniques statistiques et économétriques à l'aide du logiciel Stata (version 9).
} 
Cependant, l'hypothèse de départ du choix de la technique de sondage est liée à la disponibilité des moyens de communication, chez les enseignants. En conséquence, il convient de garder à l'esprit, à la lecture de l'analyse des résultats, que l'échantillon représente particulièrement les enseignants les plus concernés par les nouvelles technologies. C'est au sein de cette population que l'on souhaite identifier les différences d'usage.

\section{Finalités et freins à l'adoption des TIC dans l'enseignement supérieur}

L'informatisation croissante des universités françaises a conduit les enseignants à utiliser les principaux logiciels de base à des fins pédagogiques. En effet, si l'on se limite aux logiciels génériques on pourrait au moins décliner six usages de base: (1) le traitement de texte, (2) les tableurs, (3) des logiciels de base de données, (4) les présentations animées, (5) le courrier électronique et (6) l'Internet. Nous commencerons par donner un aperçu rapide concernant l'état d'usage de ces technologies avant de nous intéresser à leur intensité.

\section{Les usages des logiciels de base (traitement de texte, tableau et base de données)}

Le graphique 1 résume les résultats obtenus par notre enquête. On constate ainsi que le traitement de texte (Word, Latex...) et l'usage des tableurs (Excel...) font partie des outils usuels de l'enseignant du supérieur. En effet, la quasi-totalité de notre échantillon (96\% des enseignants) utilise un logiciel de traitement de texte et $81 \%$ mentionnent qu'ils utilisent les tableurs. 
Figure 1 - Adoption des principaux logiciels

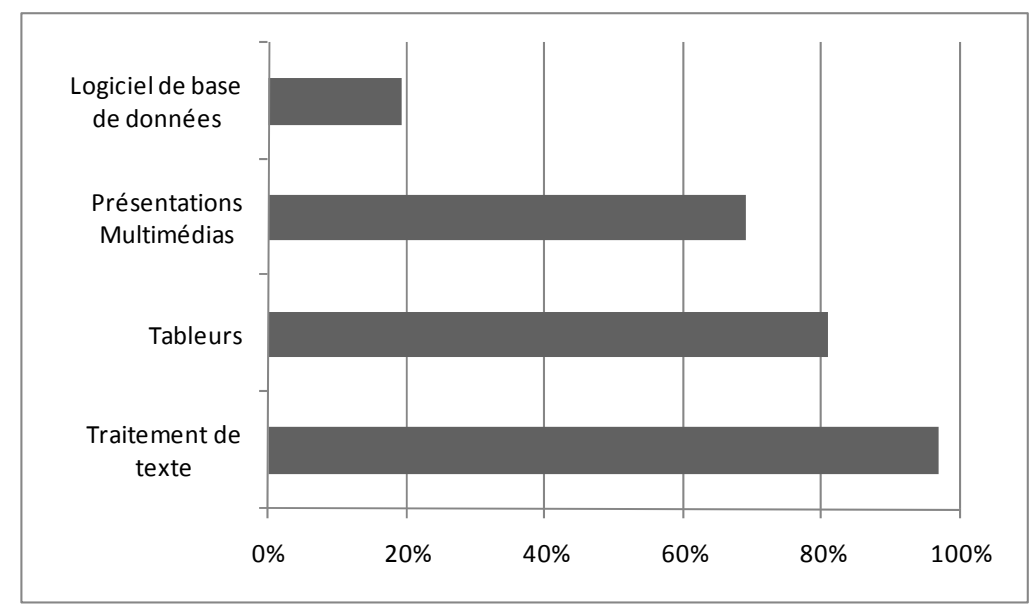

En matière d'usage des logiciels de présentations multimédia, nous constatons qu'une grande proportion d'enseignants (69\%) (Fig.1) s'oriente vers un usage régulier de cet outil dans leurs cours. Cette proportion est élevée et démontre une réorientation dans la méthode de présentation des enseignements dépassant ainsi les lectures de notes classiques. 
Figure. 2 - Formes de formation des principaux logiciels

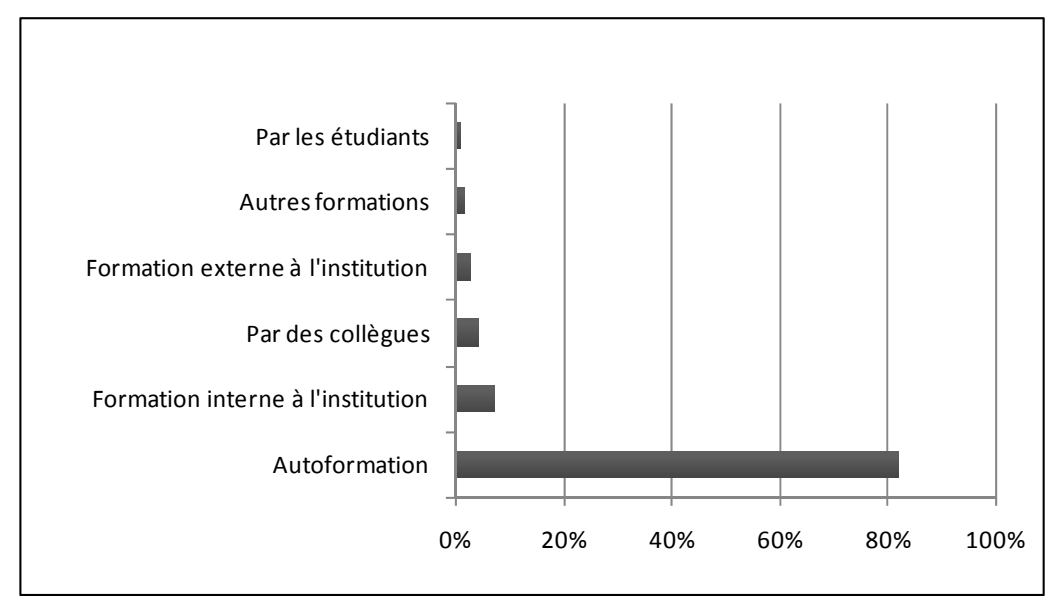

La maitrise de ces logiciels est majoritairement acquise par des processus d'autoformation. En effet, $82 \%$ des enseignants ont appris ces techniques par un apprentissage sur le tas (autoformation). Cette tendance peut s'expliquer, d'une part, par l'investissement personnel des enseignants du supérieur dans l'acquisition de savoir-faire technique essentiel (pour acquérir de nouvelles technologies) et d'autre part, par la facilité d'accès à ces logiciels. Toutefois, cette modalité d'acquisition des connaissances conduit généralement à des usages limités et mimétiques. Les apports de la technologie ne sont pas optimisés et leurs fonctionnalités ne sont pas totalement explorées. Un complément de formation nous paraît nécessaire afin de mieux structurer les usages à des fins pédagogiques.

Le constat est en revanche différent pour les logiciels de traitement des bases de données. Seulement, 19\% d'enseignants déclarent savoir utiliser les logiciels de base de données (Access, Oracle...) à des fins pédagogiques. Probablement, l'absence de formation ou d'actions de sensibilisation conduit $80 \%$ des enseignants à ne pas utiliser des logiciels d'une portée importante pour eux. 


\section{Les usages du courrier électronique et l'Internet}

La figure 3 présente une illustration des réponses apportées par les enseignants aux questions relatives à leurs usages de l'Internet et du courrier électronique. En effet, on remarque une forte utilisation, de l'ordre de $98 \%$, de l'Internet et du courrier électronique pour des finalités diverses. Cette généralisation de l'outil Internet reflète la présence d'équipements satisfaisants dans tous les lieux des répondants et une valeur d'usage significative.

Figure 3 - Adoption de l'Internet

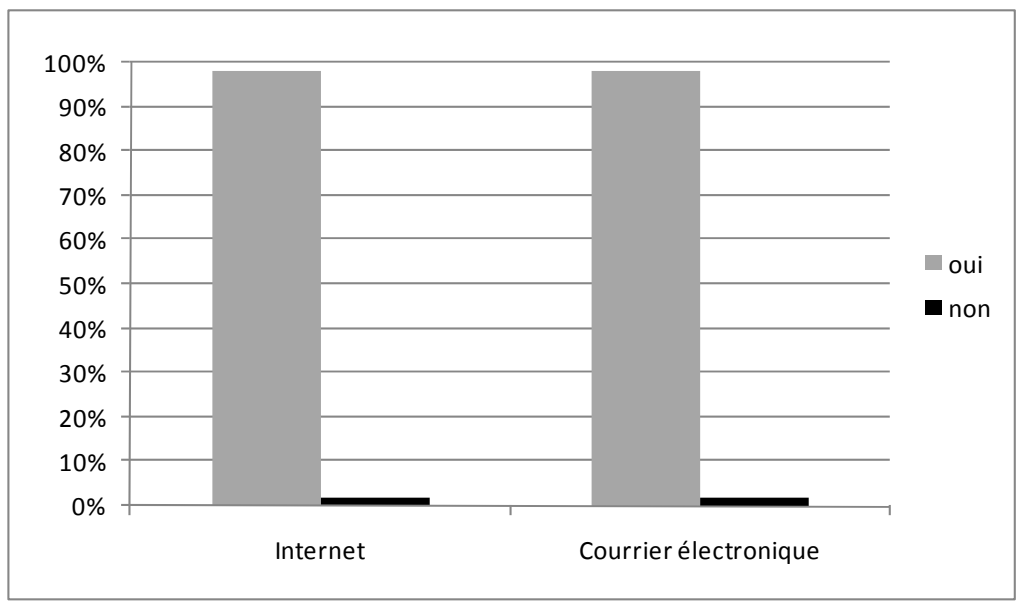

Le constat rapide dégagé de l'examen des usages des logiciels génériques montre que l'attrait qu'exercent les TIC sur les enseignants du supérieur est inégal. Alors que pour les logiciels génériques l'adoption a été rapide et généralisée, les logiciels spécifiques tels que «les logiciels des base de données » suivent des schémas d'adoption nettement plus lents. Notre étude n'a pas cherché à explorer des applications encore plus poussées. Toutefois l'hypothèse de l'existence d'une corrélation entre les usages des outils et l'investissement en formation nous paraît plausible.

\section{Les freins à l'adoption des TIC dans l'enseignement supérieur français}

De nombreuses limites et freins à l'adoption des TIC éducatives ont été mentionnés par les enseignants du supérieur. Parmi les nombreuses 
dimensions signalées (figure. 4) on peut mentionner l'absence de formation, la faiblesse de l'équipement dans les salles de cours, l'absence d'une valeur pédagogique additionnelle (perçue). Ces limites ont un impact sur l'intensité d'usage des TIC.

Figure 4-Les freins à l'usage des TIC dans les salles de cours

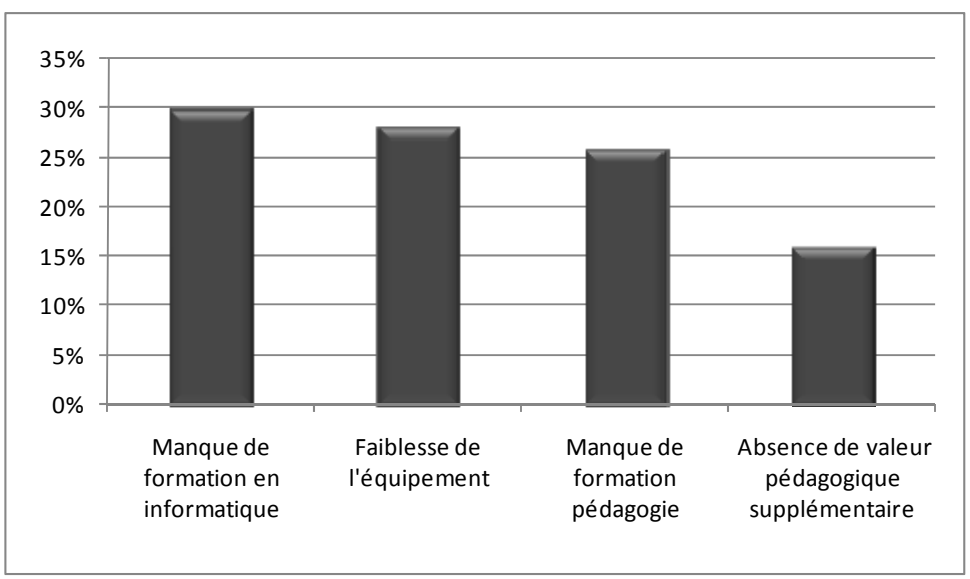

En effet, $30 \%$ des enseignants considèrent que le manque de formation en informatique pourrait bloquer leur usage en matière de TIC éducatives. L'expertise technique est indispensable à un usage généralisé et efficace. Si certains logiciels s'acquièrent facilement et ne nécessitent pas une formation poussée, d'autres logiciels spécialisés nécessitent des processus d'apprentissage importants (time-consuming) et semblent même être pénibles à utiliser.

L'absence d'équipement dans les salles de cours pèse également sur leurs usages. En effet, si l'équipement a gagné les universités, cet équipement reste concentré dans les bureaux et moins dans les salles d'enseignement, même s'il convient de noter que cette tendance tend à changer. De nombreux enseignants ont mentionné la faiblesse de moyens ou d'équipement (salles équipées en outils multimédia, ordinateurs connectés, rétroprojecteurs...) comme un frein important à la généralisation des usages.

On constate, également, que $16 \%$ des répondants à cette enquête affirment que la technologie n'apporte pas de valeur supplémentaire en comparaison à la pédagogie traditionnelle. Ce constat concernant le scepticisme de la valeur 
pédagogique est également mentionné dans d'autres études similaires à la notre comme celles de l'OCDE (2005) et de l'UNESCO (2003). En effet, les enseignants n'estiment pas nécessaire de changer les méthodes d'enseignements traditionnelles. Ceci pourrait être expliqué par un phénomène de résistance aux changements et pourrait révéler des écarts en matière d'adoption selon les disciplines.

Après avoir caractérisé les principales tendances et freins limitant les usages des TIC éducatives, notre article cherche à présent à comprendre les différences en matière d'intensité d'usage. De notre point de vue, c'est à ce niveau que l'on pourrait révéler des fractures numériques de second degré (d'usage) importantes.

\section{Description et analyse des modèles d'usage}

Afin de cerner la variété et l'intensité des usages nous avons adopté deux approches complémentaires.

La première approche consiste à calculer un score global d'usage des principales technologies (score total d'usage). Ce score est obtenu en sommant trois sous-scores. Le premier score (score en matière de communication) regroupe les usages qui répondent à un besoin de communication et qui se base sur différents outils et techniques, tels que : l'usage des Plateformes technologiques, l'usage des techniques de visioconférence, l'usage du Jumelage de correspondants, l'usage des IRC (Internet Relay Chat) et l'usage des Blogs. Le deuxième score (score en matière d'Information) regroupe les usages liés à l'acquisition d'information, tels que : la consultation et la mise à disposition des étudiants de sites Web ou de pages personnelles, l'usage des Cédérom des enseignements, l'usage des enregistrements vidéo des cours, l'usage des enregistrements audio des cours et enfin l'usage des Wikis. Enfin, le troisième score (score Collaboration et Interaction) répond à un besoin d'usage de ces technologies afin d'explorer les possibilités de collaboration et qui se résume essentiellement dans ces techniques ou moyens. Nous pouvons citer, entre autres, la mise en place de tutorat pédagogique en ligne, l'orientation du processus d'apprentissage vers des modalités collaboratives (travail en groupe à distance), l'usage des jeux de rôle par les étudiants ou encore l'usage des techniques de la simulation. 
La seconde approche a cherché à élaborer un modèle général qui tient compte de l'ensemble des dimensions d'usage. Notre démarche est inspirée des méthodologies développées par les principales institutions internationales et les instituts spécialisés (ITU). Nous avons cherché à tester une hypothèse concernant l'impact de la stabilisation des technologies sur les dynamiques d'usage. En effet, l'âge des diverses technologies et des logiciels pourrait être corrélé avec l'intensité d'usage. Ainsi, nous avons supposé que l'adoption des technologies stabilisées devrait être plus importante pour les TIC les plus anciennes comparativement aux TIC de la nouvelle génération. L'effet de la stabilisation de l'innovation et l'effet d'adoption sont supposés jouer à plein ici. Ainsi, il convient de tester des modèles où l'on accorde davantage de poids aux technologies de communication comparativement aux autres technologies. Pour illustrer cette voie de recherche, trois modèles ont été considérés ${ }^{7}$. Le modèle 5 considère les coefficients suivants : 0,5 (communication) ; 0,3 (pédagogie) et 0,2 (interactions). Le modèle 6 a pris en compte les valeurs respectives de 0,$6 ; 0,3$ et 0.1 et enfin, le modèle 7 a pris en compte les valeurs 0,$55 ; 0.3$ et 0,15 .

\section{ANALYSE DES USAGES DES TIC PAR LES ENSEIGNANTS}

Cinq caractéristiques sont apparues constituer des variables explicatives clés de l'intensité d'usage : le genre, la nature de l'emploi, la participation à des dispositifs d'enseignements à distance et l'activité de recherche. Chacune de ses variables semble exercer un effet sur l'usage et sur l'intensité d'usage des TIC. Nous proposons de les examiner tour à tour dans les paragraphes suivants.

\section{L'influence de l'âge de l'enseignant sur les usages}

La figure 5 indique une relation directe entre l'âge de l'enseignant et l'intensité d'usage des TIC. Ainsi une corrélation positive est trouvée quelle que soit la finalité considérée. Cette relation est surprenante à plus d'un titre. D'une part, de nombreux travaux montrent en général l'existence d'une courbe en cloche concernant l'investissement pédagogique de l'enseignant et son âge. L'investissement pédagogique est supposé augmenter de manière régulière et atteindre son maximum une dizaine d'années après l'entrée en

${ }^{7}$ En l'absence d'une méthodologie stabilisée, nous avons considéré des pondérations propres. 
exercice de l'enseignant. Par la suite, cet investissement est supposé diminuer. D'autre part, les résultats standards de la littérature portant sur la fracture numérique montrent la vulnérabilité des personnes âgées comparativement aux jeunes.

Notre résultat peut cependant être compris en formulant deux hypothèses complémentaires. La première concerne la contrainte de temps. Les enseignants les plus âgées semblent disposer davantage de temps pour pouvoir s'impliquer dans les dispositifs à distance. La situation familiale pourrait également être proposée comme élément explicatif. D'autre part, on pourrait supposer un effet d'apprentissage de l'usage de ces technologies par les enseignants les plus âgés ${ }^{8}$.

Figure 5 - Evolution du score moyen d'usage par tranche d'âge

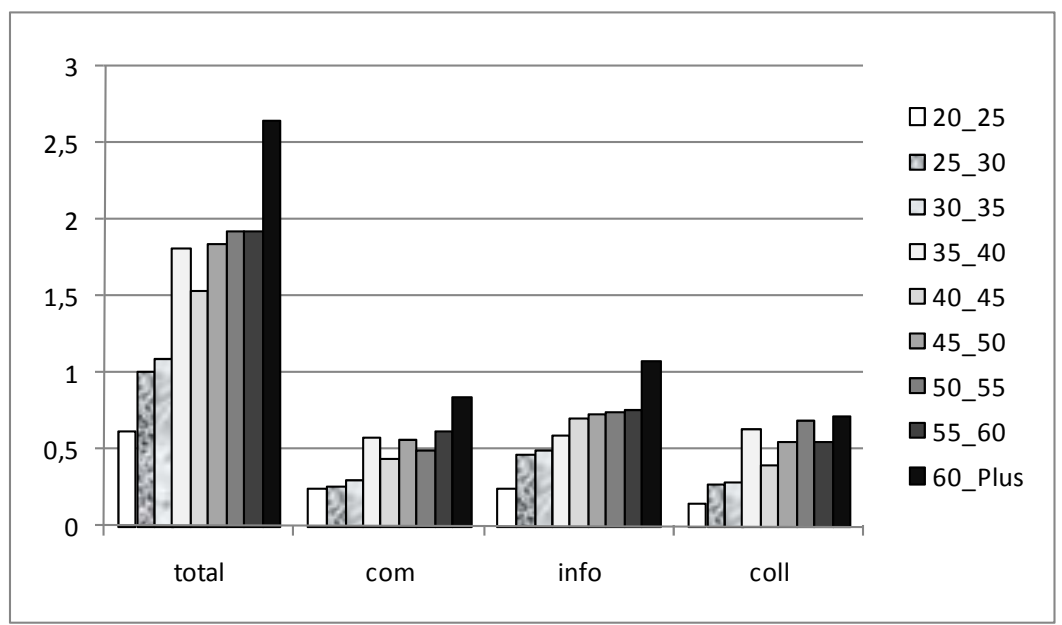

\section{L'influence du genre sur les usages}

Le graphique suivant montre que les enseignants de sexe masculin ont une intensité d'usage des TIC plus élevée que celle de sexe féminin. Ce résultat est valable pour les quatre types de scores (global, en matière de communication, en matière d'information ou en matière de collaboration).

\footnotetext{
${ }^{8}$ Ce résultat devrait être relativisé à la lumière de notre échantillon considéré.
} 
La fracture de genre en matière d'usage pour les enseignants du supérieur est également constatée au sein des entreprises et dans d'autres milieux professionnels. Cependant, un examen plus détaillé portant sur les tranches d'âge fournit davantage de détails sur ces différences.

\section{Figure 6 - Différence des scores d'usage de l'Internet selon le genre}

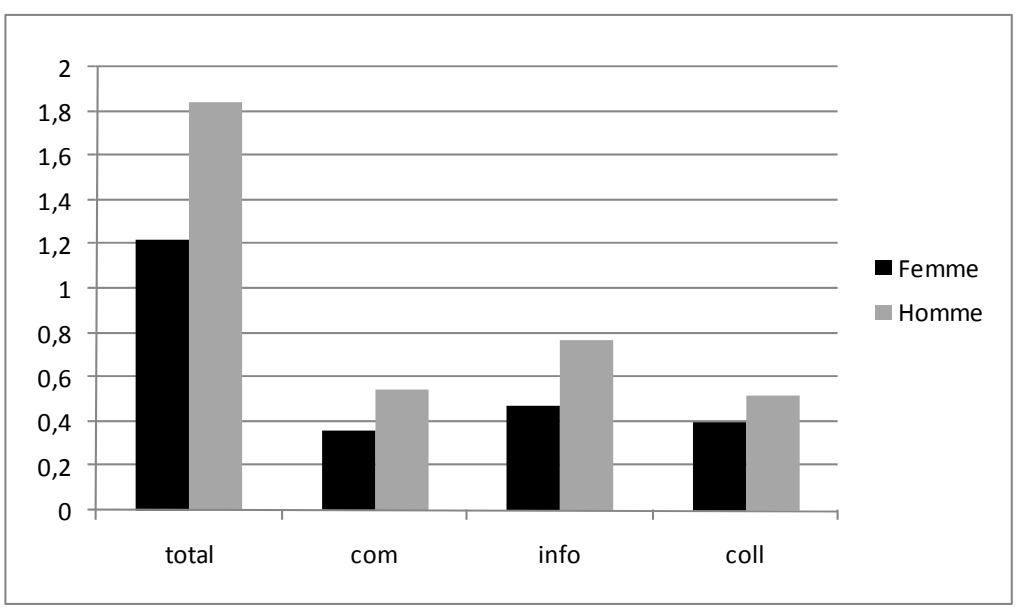

En effet on constate que ces différences atteignent leur maximum dans la tranche d'âge de 30 à 35 ans. En revanche, ce constat ne semble pas être valable pour la tranche d'âge de 60 ans et plus. Les enseignantes âgées de plus de 60 ans admettent une intensité d'usage des TIC plus importante que celle des enseignants. Il n'existe pas d'explication particulière à ce résultat ! 
Figure 7 - Différence des scores d'usage des TIC selon le genre et l'âge

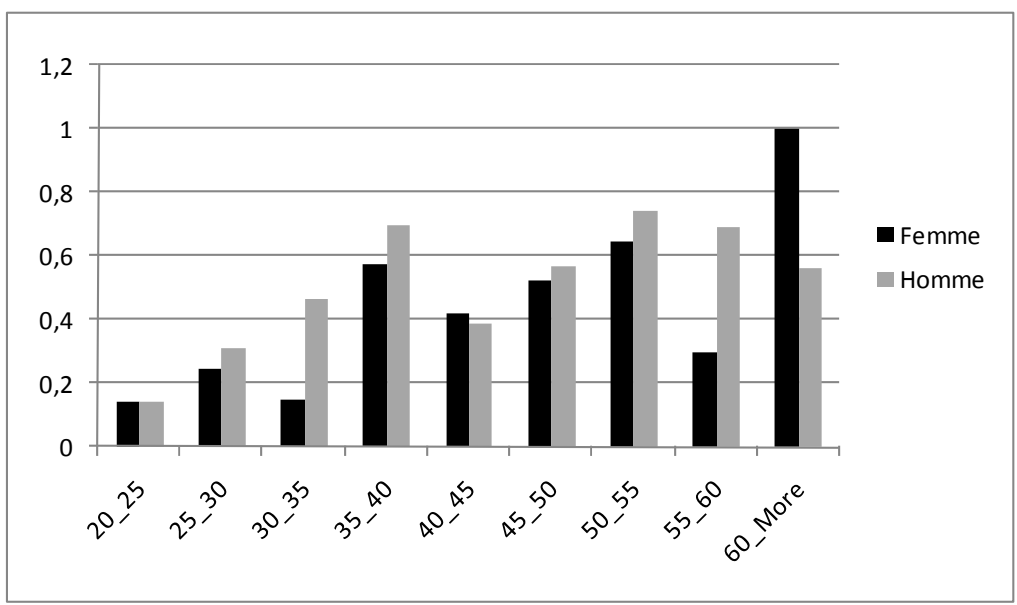

\section{L’influence de l’ancienneté sur les usages}

Pour l'ensemble des enseignants contactés, on remarque de manière nette, d'après la figure qui suit, que les jeunes enseignants (moins de 30 ans) utilisent de manière moins intensive les TIC que les autres sous-populations. Cette observation corrobore les résultats d'ANDERSSON (2006). Ceci conduit à la nécessité de prendre en compte cette observation dans les processus de formation des enseignants du supérieur. L'investissement initial dans la préparation des enseignements, la nécessité de fournir une recherche de qualité pour un avancement rapide de la carrière, les effets d'apprentissage sont autant d'éléments qui pourraient être mobilisés pour expliquer ce résultat. 
Figure 8 - Différences dans les scores d'usage des TIC en fonction de l'ancienneté

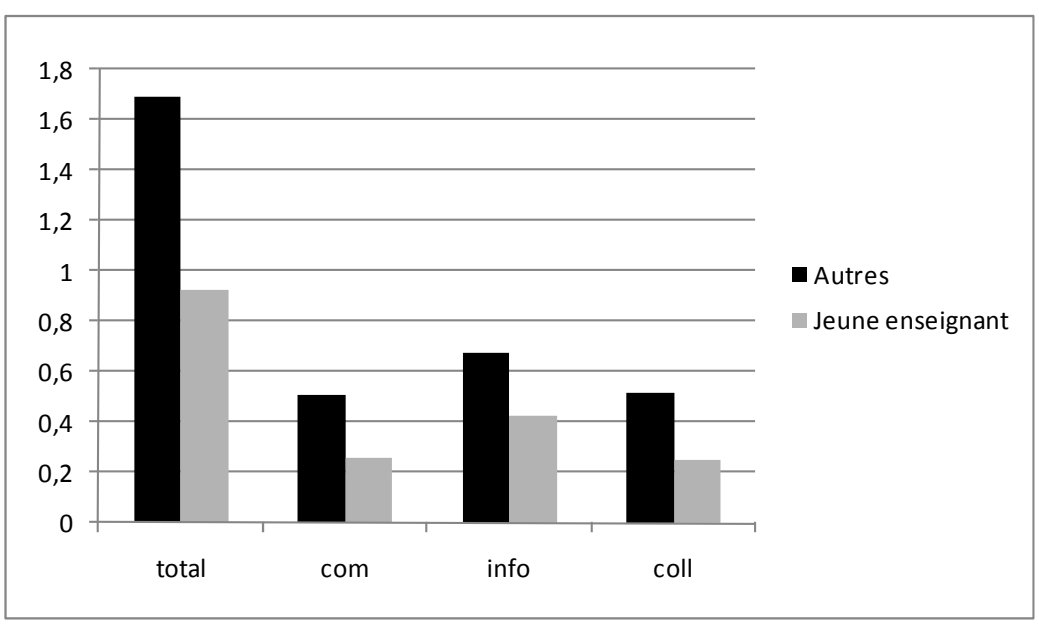

\section{L'influence du statut des enseignants sur l'intensité d'usage}

Notre enquête démontre une différence d'intensité d'usage des TIC entre les enseignants vacataires et les enseignants titulaires de leur poste. Les vacataires admettent globalement une intensité d'usage, pour divers logiciels et applications pédagogiques, moins importante que leurs homologues titulaires. Ceci peut s'expliquer en premier lieu par les modalités d'attribution du matériel informatique. En effet, on constate que le matériel informatique est majoritairement financé par les institutions auxquelles appartiennent les enseignants titulaires, alors qu'il est en général pris en charge directement par les vacataires. Cette disparité dans l'acquisition du matériel informatique joue un effet négatif sur les usages. En second lieu, les enseignants titulaires ont une probabilité de disposer d'un poste de travail attitré (bureau équipé d'un ordinateur) plus élevée que les enseignants vacataires. Ces derniers, le plus souvent disposent d'un poste partagé (poste multi-utilisateurs). Ceci affecte fortement l'intensité d'usage. L'effet d'ancienneté précédemment mentionné joue également à plein. La moyenne 
d'âge des enseignants non-titulaires de notre échantillon est de 33 ans alors que celle des titulaires atteint 43 ans.

\section{Intensité d'usage des TIC et participation à un dispositif de cyber- formation}

L'intensité d'usage des TIC est également affectée par la participation des enseignants à des dispositifs d'enseignements à distance. Dans notre échantillon, cette proportion ne représente que 20\% (totalement ou partiellement en dispositif de e-Learning). En effet, comme le montre le tableau infra, on constate une corrélation positive et croissante entre l'implication dans un dispositif de e-Learning et l'intensité d'usage des TIC. La participation à un dispositif à distance via l'Internet permet aux enseignants d'explorer des possibilités technologiques non forcément utilisées dans les dispositifs face-à-face et permet d'accroître significativement leur intensité d'usage. Un transfert des applications explorées dans les dispositifs en ligne vers les enseignements traditionnels a lieu par la suite. L'implication des enseignants dans des dispositifs d'enseignement en ligne pourrait constituer un levier important de décollage des usages.

Tableau 1. e-Learning et intensité d'usage des TIC

\begin{tabular}{|l|l|}
\hline $\begin{array}{l}\text { Pourcentage d'enseignement en } \\
\text { ligne }\end{array}$ & $\begin{array}{l}\text { Moyenne de l'intensité } \\
\text { d'usage }\end{array}$ \\
\hline $0 \%$ & $\mathbf{1 , 1 4}$ \\
Moins de $50 \%$ & $\mathbf{2 , 7 3}$ \\
$50 \%$ & $\mathbf{3 , 5}$ \\
Plus de $50 \%$ & $\mathbf{4 , 8 7}$ \\
$\mathbf{1 0 0} \%$ & $\mathbf{5 , 6}$ \\
\hline
\end{tabular}

\section{L’impact de l'activité de recherche sur les usages des TIC}

L'activité de recherche semble exercer un effet positif sur l'intensité d'usage des TIC. En effet, l'intensité d'usage des chercheurs confirmés et experts dépasse largement l'intensité d'usage des non-chercheurs. En revanche, l'intensité d'usage des jeunes chercheurs et des chercheurs juniors est plus 
faible que celle des chercheurs confirmés. Ceci pourrait être expliqué par une allocation du temps en faveur de la recherche au détriment des activités d'enseignement dans un premier stade. D'autre part, elle peut s'expliquer par un effet négatif de l'ancienneté comme nous l'avons précédemment souligné. L'activité de recherche semble permettre aux enseignantschercheurs d'explorer les possibilités technologiques qui sont alors transférées dans le processus d'enseignement.

Figure 9 - Différences dans les scores d'usage des TIC en fonction du grade de recherche

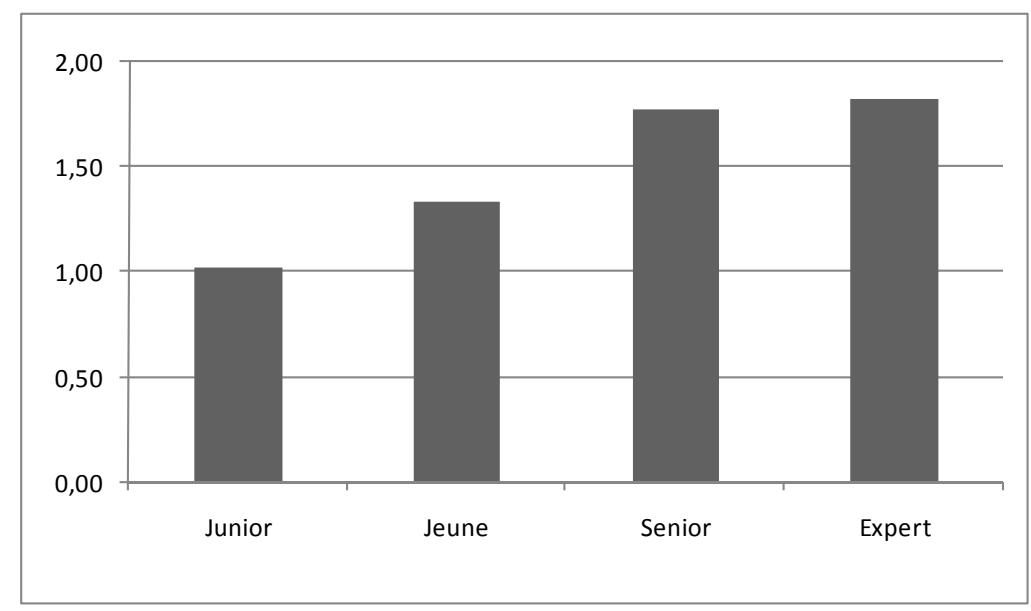

L'analyse descriptive selon les scores d'intensité d'usage nous a permis de caractériser des différences importantes entre les groupes d'enseignants. Ainsi, nous avons montré de manière significative que le degré d'usage des TIC varie selon les caractéristiques de l'enseignant. Dans la section suivante, nous proposons d'approfondir cette analyse descriptive par une étude économétrique.

\section{ANALYSE ECONOMETRIQUE DES USAGES DES TIC}

Afin d'étudier les facteurs qui influencent le degré d'usage des TIC par les enseignants du supérieur nous avons emprunté un modèle économétrique de type probit ordonné. La finalité consiste à examiner la probabilité de l'usage des TIC selon diverses caractéristiques de l'enseignant (sexe, âge, grade, activité de recherche...) ainsi qu'en fonction de son environnement du 
travail (utilisation de l'Internet en cours, possession d'un PC sur le lieu de travail...).

Ce modèle général est également décomposé en trois sous-modèles se distinguant par les finalités d'usage des TIC mentionnées auparavant. Le modèle 2 détaille l'intensité d'usage des TIC à but de communication; le modèle 3 détaille l'intensité d'usage des TIC à but d'informationnel; et enfin, le modèle 4 examine l'intensité d'usage à but de collaboration et d'interaction.

\section{La structure générale du modèle}

Les variables de base de notre étude sont des variables qualitatives binaires. L'enseignant répond en général à des questions en matière d'usage par oui ou non. Cette manière de procéder permet de qualifier la variable de qualitative binaire (elle prend la valeur 1 si l'enseignant utilise la technologie (réponse oui) et la valeur 0 (si non). Le score global est obtenu en additionnant les trois sous scores. Ainsi se manifeste le caractère multinomial de la variable. Ceci nous autorise à recourir à un modèle de type probit ordonné. En effet, cette méthode permet d'étudier l'influence exercée par une série de facteurs sur une variable multinomiale ordonnée (Greene, 2000 ; thomas, 2000).

Les modèles probit ordonnés sont généralement des méthodes basées sur la vraisemblance. Le modèle latent est similaire à celui d'un probit binomial :

$$
y_{i}^{*}=\beta x_{i}+\varepsilon_{i}
$$

$x_{\mathrm{i}}$ désigne un vecteur de variables endogènes, $\beta$ désigne un vecteur de paramètres et $\varepsilon_{i}$ est considérée comme l'erreur résiduelle qui suit une distribution normale. Dans le cas du modèle probit multinomial ordonné, on observe :

$$
y_{i}=j \text { si } c_{j}<y_{i}^{*}<c_{j+i}
$$

$j=0,1, \ldots, J$ représente les différentes modalités de la variable endogène. Les probabilités associées à ces différentes modalités sont calculées de la manière suivante : 


$$
\operatorname{Pr} o b\left(y_{i}=j\right)=\Phi\left(c_{j+1}-\beta x_{i}\right)-\Phi\left(c_{j}-\beta x_{i}\right)
$$

Avec $\Phi$ représente la fonction de distribution de la loi normale.

L'ajustement du modèle se fait par la méthode du maximum de vraisemblance (MADDALA et FLORES, 2001), cette dernière s'écrivant suivante :

$$
L=\prod_{i=1}^{N} \prod_{j=0}^{J} F_{i j}(x, \beta)^{y_{i j}}
$$

\section{Les variables}

\section{Les variables dépendantes : mode d'usage des TIC}

Les variables dépendantes utilisées dans les quatre modèles sont décrites par les scores détaillés auparavant.

La première variable (score en matière de communication) regroupe les usages qui répondent à un besoin de communication qui se base sur différents outils et techniques, tels que l'usage des plateformes, l'usage des techniques de visioconférence, l'usage du jumelage de correspondants, l'usage de l'IRC (Internet Relay Chat) et l'usage des Blogs (soit cinq types d'usage). Le score varie de 0 à 5 .

La deuxième variable (score en matière d'Information) regroupe les usages liés à l'acquisition de l'information, tels que la consultation des sites Web ou des pages personnelles, l'emploi de Cédérom de cours, l'usage d'enregistrements vidéo des cours et l'usage des enregistrements audio des cours. Le score varie de 0 à 4 .

La troisième variable (score Collaboration/Interaction) répond à un besoin de collaboration et se résume essentiellement dans l'usage des techniques ou des moyens suivants : l'emploi d'un tutorat pédagogique en ligne, l'apprentissage collaboratif, l'usage de jeu de rôle par l'étudiant ou le professeur, l'usage de simulation et la mise en place de Wikis. Le score varie ici de 0 à 5 . 
Enfin, la quatrième variable (score Total) regroupe l'ensemble des usages des TIC, quel que soit le besoin, qui sont au nombre de quatorze. Elle intègre donc l'ensemble des outils et techniques inclus dans les trois premières variables. Le score varie de 0 à 14 .

Chacune des variables est décrite de la manière suivante:

$$
\left\{\begin{array}{l}
y_{i}=0 \quad \text { si zéro usage } \\
y_{i}=1 \quad \text { si un seul type d'usage } \\
y_{i}=2 \quad \text { si deux types d'usage } \\
\vdots \\
y_{i}=n \quad \text { si n type d'usage }
\end{array}\right.
$$

Avec, $n=5$ pour le score de communication, $n=4$ pour le score Information/Pédagogie, $n=5$ pour le score Collaboration/Interaction et $n=14$ pour le score Total. 
Tableau 2. Définitions des variables dépendantes

\begin{tabular}{llc}
\hline \multicolumn{1}{c}{ Variable } & \multicolumn{1}{c}{ Définition } & $\begin{array}{c}\text { Nombre } \\
\text { d'usage }(n)\end{array}$ \\
\hline Score Communication & $\begin{array}{l}\text { Les usages liés à un besoin de } \\
\text { communication }\end{array}$ & 5 \\
$\begin{array}{l}\text { Score } \\
\text { Information/Pédagogie }\end{array}$ & $\begin{array}{l}\text { Les usages liés à l'acquisition de } \\
\text { l'information }\end{array}$ & 4 \\
$\begin{array}{c}\text { Score } \\
\text { Collaboration/Interaction }\end{array}$ & $\begin{array}{l}\text { Les usages liés à un besoin de } \\
\text { collaboration }\end{array}$ & 4 \\
\hline Score Total & Tous les usages & 14 \\
\hline
\end{tabular}

\section{Les variables explicatives liées aux caractéristiques de l'enseignant}

Les variables utilisées portant sur les caractéristiques de l'enseignant sont au nombre de six. D'une part, le genre (homme, femme), d'autre part, l'âge décomposé en neuf classes : 20-25, 25-30,.., 60 ans et plus), ensuite, le statut de l'enseignant (titulaire, vacataire, professionnel...). Par ailleurs nous avons considéré que le taux d'enseignement ou de cours effectué à distance appartient aux catégories suivantes $(0 \%, 0-50 \%, 50 \%, 50-100 \%$ et $100 \%)$. Enfin, le grade de recherche des enseignants-chercheurs a été décomposé en 4 catégories (chercheur junior, jeune chercheur, chercheur senior ou chercheur expert).

\section{Les variables explicatives liées à l'environnement du travail de l'enseignant}

L'environnement du travail de l'enseignant du supérieur est résumé ici par diverses variables : la possession d'un ordinateur (sur le lieu de travail et à domicile), l'accès à l'Internet en cours, la présence de matériel informatique en cours et le nombre d'étudiants qui suivent les cours durant l'année universitaire (2005/2006). Initialement d'autres variables ont été considérées 
mais compte tenu du manque de réponses nous les avons exclues dans les traitements économétriques.

Tableau 3. Définitions des variables explicatives

\begin{tabular}{|c|c|}
\hline $\begin{array}{c}\text { Variables } \\
\text { Explicatives }\end{array}$ & Définitions \\
\hline \multicolumn{2}{|c|}{ I. Caractéristiques de l'enseignant } \\
\hline Sexe & $\begin{array}{l}\text { Variable binaire décrivant le sexe de l'enseignant } \\
\text { répondant: égale à : } \\
\qquad 1 \text { si sexe Masculin } \\
0 \text { si sexe Féminin }\end{array}$ \\
\hline Age & $\begin{array}{l}\text { Variable continue répartie sur } 9 \text { tranches d'âge de } 5 \\
\text { ans chacune : } \\
20-25 ; 25-30 ; \ldots ; 60 \text { ans et plus }\end{array}$ \\
\hline Emploi & $\begin{array}{l}\text { Variable qualitative décrivant le statut de } \\
\text { l'enseignant: répartie selon } 3 \text { modalités: } \\
\text { 1- Titulaire } \\
\text { 2- Vacataire } \\
\text { 3- } \text { Autres Situations }\end{array}$ \\
\hline $\begin{array}{l}\text { Enseignement à } \\
\text { Distance }\end{array}$ & $\begin{array}{l}\text { Le taux (\%) d'enseignement ou de cours effectué à } \\
\text { distance : répartie selon } 5 \text { modalités : } \\
\text { 1. } 0 \% \text {; } \\
\text { 2. } 0 \%--50 \% ; \\
\text { 3. } 50 \% \\
\text { 4. } 50 \%-100 \% ; \\
\text { 5. } 100 \%\end{array}$ \\
\hline Grade de recherche & $\begin{array}{l}\text { Le grade de recherché de l'enseignant: décomposé } \\
\text { en } 4 \text { catégories } \\
\text { 1. chercheur junior ; } \\
\text { 2. jeune chercheur; } \\
\text { 3. chercheur senior; } \\
\text { 4. chercheur expert }\end{array}$ \\
\hline \multicolumn{2}{|c|}{ II. Environnement du travail de l'enseignant } \\
\hline Ordinateur au travail & $\begin{array}{l}\text { Variable binaire égale à : } \\
1 \text { si l'enseignant dispose d'un ordinateur sur } \\
\text { son lieu de travail } \\
0 \text { si non }\end{array}$ \\
\hline $\begin{array}{l}\text { Ordinateur à } \\
\text { domicile }\end{array}$ & $\begin{array}{l}\text { Variable binaire égale à : } \\
1 \text { si l'enseignant dispose d'un ordinateur à }\end{array}$ \\
\hline
\end{tabular}




\begin{tabular}{|l|l|}
\hline & $\begin{array}{l}\text { domicile } \\
0 \text { si non }\end{array}$ \\
\hline Internet en cours & $\begin{array}{l}\text { Variable binaire égale à : } \\
1 \text { si l'enseignant a accès à Internet en cours } \\
0 \text { si non }\end{array}$ \\
\hline $\begin{array}{l}\text { Equipements } \\
\text { informatiques en sale } \\
\text { de cours }\end{array}$ & $\begin{array}{l}\text { Variable binaire égale à : } \\
1 \text { si les salles de cours sont équipées en } \\
\text { matériel informatique } \\
0 \text { si non }\end{array}$ \\
\hline Nombre d'étudiants & $\begin{array}{l}\text { Le nombre approximatif des étudiants qui suivent les } \\
\text { cours de l'enseignant répondant durant l'année en } \\
\text { cours. }\end{array}$ \\
\hline
\end{tabular}

\section{Les résultats économétriques}

Les tableaux suivants résument nos résultats économétriques. La liaison ou la dépendance entre l'usage des TIC par les enseignants du supérieur, identifiée par les quatre scores prédéfinis, et les caractéristiques ou l'environnement de travail, est exposée sous forme de quatre modèles différents ${ }^{9}$.

L'absence d'un dispositif de formation généralisé à l'ensemble des enseignants est criante. Notamment en ce qui concerne les jeunes enseignants. Il convient à ce niveau de souligner que le recrutement des jeunes maîtres de conférences s'effectue faiblement sur leurs qualités pédagogiques et qu'aucune formation particulière n'est dispensée, notamment en matière de TIC. Ainsi, au début de sa carrière le jeune enseignant n'a aucune stratégie éducative particulière sur laquelle pourrait être greffée l'emploi des TIC.

\section{L'effet des caractéristiques de l'enseignant sur l'usage des TIC}

\footnotetext{
${ }^{9}$ Il convient de garder à l'esprit, lors de la lecture des résultats, que les quatre modèles sont estimés séparément. Ni les coefficients ni leurs significativités ne sont dépendants les uns des autres. Autrement dit, il est possible d'obtenir des coefficients élevés dans les trois derniers modèles alors que celui qui concerne le modèle globale peut être faible. Aussi, nous pouvons avoir des coefficients statistiquement significatifs dans les modèles des sous-scores (Modèle 1 , Modèle 2 et Modèle 3 ) et qui sont non significatifs pour le modèle du score total.
} 
Les résultats économétriques corroborent les résultats obtenus par l'analyse descriptive. En effet, il convient de constater que les enseignants de sexe masculin ont une intensité à utiliser les TIC à but de communication, d'information et de collaboration plus élevée que celles des enseignantes. Le tableau des résultats montre néanmoins une légère accentuation de cette différence. En effet, les coefficients de la variable sexe, qui reflète ici le sexe masculin, sont positifs et statistiquement significatifs. Ces premiers éléments permettent de mettre en évidence une certaine fracture de genre dans le processus d'intensité d'usage des TIC par les enseignants du supérieur.

Concernant l'âge des enseignants, il apparaît que son influence sur les usages diffère selon les modèles. On remarque que les enseignants âgés de 30 ans et plus sont davantage intéressés par l'usage des TIC à des fins pédagogiques que les autres. Par contre, comme l'a démontré ANDERSSON (2006) en Suède, les jeunes enseignants, de moins de 30 ans, utilisent faiblement ces technologies dans un objectif pédagogique. Nos estimations permettent de montrer que l'âge a un effet significatif sur l'intensité d'usage à des finalités d'information. En revanche, l'usage des TIC par les enseignants universitaires, à des besoins de communication, n'a aucune liaison ou corrélation avec leurs âges.

La titularisation de l'enseignant a un effet positif sur son degré d'usage des TIC, quelque soit la finalité de cette utilisation. Ce résultat corrobore les constatations déduites de l'analyse descriptive. Ceci peut s'expliquer essentiellement par les modalités d'attribution du matériel informatique, qui joue un effet négatif sur les usages. Les enseignants titulaires ont une probabilité de disposer d'un poste de travail attitré (bureau équipé d'un ordinateur) plus élevée que les enseignants vacataires.

Le tableau 4 montre que le degré d'usage des TIC par les enseignants augmente selon leurs avancements dans leurs recherches. Les scores augmentent avec le grade de recherche. En effet, on remarque que les nonchercheurs et les chercheurs juniors admettent des intensités d'usage des TIC moindre que les autres catégories. On constate également que les jeunes chercheurs utilisent davantage les TIC pour répondre à des besoins de collaboration et d'interaction. En revanche, les chercheurs séniors utilisent plus les TIC à des finalités pédagogiques et informationnelles. Ceci confirme l'idée selon laquelle l'activité de recherche semble permettre aux enseignants-chercheurs d'explorer les possibilités technologiques qui sont alors transférées dans le processus d'enseignement et de pédagogie. 
En ce qui concerne l'enseignement à distance, on constate que l'implication dans une activité en ligne exerce une influence positive et significative sur l'intensité globale d'usage des TIC par les enseignants du supérieur. En conséquence, plus le taux ou la part du cours dispensée par l'enseignant en ligne augmente et plus son intensité d'usage des TIC augmente. Ce résultat témoignerait de la place importante occupée par les TIC en tant qu'outil de communication, d'information et de collaboration pour les enseignants à distance.

\section{L'effet de l'environnement du travail de l'enseignant sur l'usage des TIC}

Relativement à l'influence de l'environnement du travail de l'enseignant universitaire sur son usage des TIC, les résultats des quatre modèles indiquent que l'usage de l'Internet en cours a une influence positive et significative quelles que soient les finalités. De même, on observe que la possession d'un ordinateur sur le lieu du travail a un effet positif sur l'usage des TIC par les enseignants à des finalités pédagogiques. Le coefficient de cette variable (Ordinateur au travail) est positif et significatif dans le modèle 3. Ceci confirme le résultat dégagé dans l'analyse descriptive.

Par ailleurs, on constate également que l'enseignant dispensant ses cours dans une salle équipée en matériel informatique (tels que l'ordinateur, la connexion Internet, les outils multimédias et l'équipement en vidéoprojecteur) a un degré d'usage des TIC plus important que les autres.

Enfin, la variable nombre d'étudiants suivant les cours, a un effet positif sur l'intensité d'usage des TIC par les enseignants du supérieur, mais cette influence demeure assez faible. Il convient de remarquer que cette variable (nombre d'étudiant enseignés) a un effet positif et significatif sur le degré d'usage des TIC par les enseignants à des finalités de communications. Ceci peut être expliqué par le fait que lorsque le nombre d'étudiants en classe augmente la communication face à face avec ces derniers devient difficile à gérer. Ceci les pousse à davantage communiquer par les TIC et surtout par les courriels. 


\section{Les résultats de la seconde approche}

Concernant les résultats de la seconde approche visant à tenir compte de l'ensemble des dimensions d'usage et à tester l'impact de la stabilisation des technologies sur les dynamiques d'usage, l'âge des diverses technologies et des logiciels est corrélé avec l'intensité d'usage. Ainsi, nous avons supposé que l'adoption des technologies stabilisées devrait être plus grande pour les TIC les plus anciennes comparativement aux TIC de la nouvelle génération. Nous avons testé des modèles où l'on accorde davantage de poids aux technologies de communication comparativement aux autres technologies.

La lecture du tableau 5 nous amène à constater qu'il n'existe pas de différences notables entre les résultats du premier modèle et ceux des trois nouveaux, dans lesquels nous avons attribué différentes pondérations. En revanche, le seul point de divergence apparaît au niveau de la variable " possession d'un ordinateur à domicile». 
Tableau 4. Les déterminants de l'intensité d'usages des TIC par les enseignants du supérieur

\begin{tabular}{|c|c|c|c|c|}
\hline \multirow[b]{2}{*}{$\begin{array}{l}\text { Variables } \\
\text { explicatives }\end{array}$} & \multicolumn{4}{|c|}{$\begin{array}{c}\text { Variables dépendantes } \\
\text { Scores }\end{array}$} \\
\hline & $\begin{array}{c}\text { Modèle } 1 \\
\text { Total }\end{array}$ & $\begin{array}{c}\text { Modèle } 2 \\
\text { Communication }\end{array}$ & $\begin{array}{c}\text { Modèle } 3 \\
\text { Information } \\
\text { / Pédagogie }\end{array}$ & $\begin{array}{c}\text { Modèle } 4 \\
\text { Collaboration/ } \\
\text { Interaction }\end{array}$ \\
\hline \multicolumn{5}{|c|}{ Caractéristiques de l'enseignant } \\
\hline $\begin{array}{l}\text { Sexe : } \\
\text { Femme } \\
\text { Homme }\end{array}$ & $\begin{array}{c}\text { Réf. } \\
0,2423 * *\end{array}$ & $\begin{array}{l}\text { Réf. } \\
0,2222 *\end{array}$ & $\begin{array}{l}\text { Réf. } \\
0,2812 * *\end{array}$ & $\begin{array}{l}\text { Réf. } \\
0,0542\end{array}$ \\
\hline $\begin{array}{l}\text { Age : } \\
20-25 \text { ans } \\
25-30 \text { ans } \\
30-35 \text { ans } \\
35-40 \text { ans } \\
40-45 \text { ans } \\
45-50 \text { ans } \\
50-55 \text { ans } \\
55-60 \text { ans } \\
60 \text { ans et plus }\end{array}$ & $\begin{array}{l}\quad \text { Réf. } \\
0,1812 \\
0,4626 \\
0,6921 * * \\
0,5347 \\
0,8116 * * \\
0,5446 \\
0,6528 * \\
08082 * *\end{array}$ & \begin{tabular}{r}
\multicolumn{1}{c}{ Réf. } \\
$-0,2059$ \\
0,0980 \\
0,4221 \\
0,3146 \\
0,6354 \\
0,2403 \\
0,4307 \\
07298
\end{tabular} & $\begin{array}{cc}\text { Réf. } & \\
0,5291 & \\
0,9849 & * * \\
0,8664 & * * \\
0,8745 & * * \\
0,8929 & * * \\
0,8651 & * * \\
0,9221 & * * \\
1,0326 & * *\end{array}$ & $\begin{array}{l}\text { Réf. } \\
0,3383 \\
0,4377 \\
0,8332 * \\
0,5103 \\
0,8915 * \\
0,7541 \\
0,6023 \\
0,8199\end{array}$ \\
\hline $\begin{array}{l}\text { Emploi : } \\
\text { Vacataire } \\
\text { Titulaire } \\
\text { Autres }\end{array}$ & $\begin{array}{l}\text { Réf. } \\
0,6177 * * \\
0,4317 * * *\end{array}$ & $\begin{array}{l}\text { Réf. } \\
0,3801 \\
0,3705 * *\end{array}$ & $\begin{array}{c}\text { Réf. } \\
0,7027 * * * \\
0,5162 * * *\end{array}$ & $\begin{array}{c}\text { Réf. } \\
0,3840 \\
0,1946\end{array}$ \\
\hline $\begin{array}{l}\text { Enseignement } \\
\text { distance : } \\
0 \% \\
0-50 \% \\
50 \% \\
50-100 \% \\
100 \%\end{array}$ & $\begin{array}{l}\text { Réf. } \\
0,8750 * * * \\
1,4841 * * * \\
1,4497 * * * \\
1,9877 * * *\end{array}$ & $\begin{array}{l}\text { Réf. } \\
0,6125 * * * \\
1,6187 * * * \\
1,4834 * * * \\
1,1397 * *\end{array}$ & $\begin{array}{l}\text { Réf. } \\
0,5740 * * * \\
0,2412 \\
1,3102 * * * \\
1,1789 * *\end{array}$ & $\begin{array}{l}\text { Réf. } \\
0,9068 * * * \\
1,6160 * * * \\
0,3886 \\
1,9962 * * *\end{array}$ \\
\hline $\begin{array}{l}\text { Grade de } \\
\text { recherche : } \\
\text { Non chercheur } \\
\text { Junior }\end{array}$ & $\begin{array}{r}\text { Réf. } \\
-0,1557\end{array}$ & $\begin{array}{c}\text { Réf. } \\
-0,1243\end{array}$ & $\begin{array}{l}\text { Réf. } \\
-0,0089\end{array}$ & $\begin{array}{c}\text { Réf. } \\
-0,1101\end{array}$ \\
\hline
\end{tabular}




\begin{tabular}{|l|l|l|l|l|}
\hline Jeune & $0,2775^{* * *}$ & 0,2767 & 0,0157 & $0,3370^{*}$ \\
Senior & 0,1652 & $-0,0913$ & $0,2861^{*}$ & 0,1511 \\
Expert & 0,0802 & $-0,5252$ & 0,4336 & $-0,2710$ \\
\hline
\end{tabular}

Environnement du travail

\begin{tabular}{|c|c|c|c|c|}
\hline $\begin{array}{l}\text { ordinateur au } \\
\text { travail : } \\
\text { Non } \\
\text { Oui }\end{array}$ & $\begin{array}{c}\text { Réf. } \\
0,1047\end{array}$ & $\begin{array}{c}\text { Réf. } \\
-0,3175\end{array}$ & $\begin{array}{l}\text { Réf. } \\
0,7330 * *\end{array}$ & $\begin{array}{c}\text { Réf. } \\
-0,1540\end{array}$ \\
\hline $\begin{array}{l}\text { ordinateur à } \\
\text { domicile : } \\
\text { Non } \\
\text { Oui }\end{array}$ & $\begin{array}{c}\text { Réf. } \\
-0,0727\end{array}$ & $\begin{array}{c}\text { Réf. } \\
-0,3007\end{array}$ & $\begin{array}{c}\text { Réf. } \\
0,0445\end{array}$ & $\begin{array}{c}\text { Réf. } \\
-0,1602\end{array}$ \\
\hline $\begin{array}{l}\text { Internet en } \\
\text { cours : } \\
\text { Non } \\
\text { Oui }\end{array}$ & $\begin{array}{c}\text { Réf. } \\
0,6406^{* * *}\end{array}$ & $\begin{array}{l}\text { Réf. } \\
0,4830 * * *\end{array}$ & $\begin{array}{c}\text { Réf. } \\
0,5098 * * *\end{array}$ & $\begin{array}{c}\text { Réf. } \\
0,5502 * * *\end{array}$ \\
\hline $\begin{array}{l}\text { Equipements } \\
\text { informatiques } \\
\text { en salle de } \\
\text { cours : } \\
\text { Non } \\
\text { Oui }\end{array}$ & $\begin{array}{c}\text { Réf. } \\
0,6031^{* * *}\end{array}$ & $\begin{array}{l}\text { Réf. } \\
0,3979 * * *\end{array}$ & $\begin{array}{c}\text { Réf. } \\
0,5565^{* * *}\end{array}$ & $\begin{array}{c}\text { Réf. } \\
0,6138^{* * *}\end{array}$ \\
\hline $\begin{array}{l}\text { Nombre } \\
\text { d'étudiants } \\
\text { enseignés }\end{array}$ & 0,0001 & $0,0004 *$ & $-0,0001$ & 0,0001 \\
\hline $\begin{array}{l}\text { log likelihood } \\
\text { LR chi2 (25) }\end{array}$ & $\begin{array}{c}-766,0419 \\
231,14\end{array}$ & $\begin{array}{c}-396,1093 \\
133,96 \\
\end{array}$ & $\begin{array}{c}-477,7544 \\
153,72 \\
\end{array}$ & $\begin{array}{c}-393,0631 \\
157,13 \\
\end{array}$ \\
\hline
\end{tabular}

Réf. : variable de référence,

* coefficient significatif au seuil de $10 \%$, ** coefficient significatif au seuil de $5 \%, * * *$ coefficient significatif au seuil de $1 \%$.

Tableau 5. Estimation de l'intensité d'usage des TIC selon la deuxième approche 


\begin{tabular}{|c|c|c|c|c|}
\hline \multirow{2}{*}{$\begin{array}{r}\text { Variables } \\
\text { dépendantes }\end{array}$} & Probit & \multicolumn{3}{|c|}{ Moindres Carrées Ordinaire (MCO) } \\
\hline & $\begin{array}{c}\text { Modèle } 1 \\
\begin{array}{c}\text { Usage }=\text { com } \\
+ \text { coll } \\
+ \text { inf }\end{array}\end{array}$ & 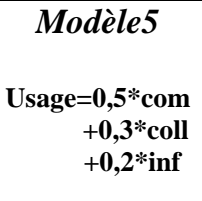 & $\begin{array}{c}\text { Modèle } 6 \\
\begin{array}{c}\text { Usage }=0,6 * \operatorname{com} \\
+0,3^{*} \operatorname{coll} \\
\\
+0,1 * \text { inf }\end{array}\end{array}$ & $\begin{array}{c}\text { Modèle } 7 \\
\begin{array}{c}\text { Usage }=0,55 * \text { com } \\
+0,3 * \text { coll } \\
\\
+0,15 * \text { inf }\end{array}\end{array}$ \\
\hline \multicolumn{5}{|c|}{ Caractéristiques de l'enseignant } \\
\hline $\begin{array}{l}\text { Sexe : } \\
\text { Femme } \\
\text { Homme }\end{array}$ & $\begin{array}{l}\text { Réf. } \\
0,2423 * *\end{array}$ & $\begin{array}{l}\text { Réf. } \\
0,0993 * *\end{array}$ & $\begin{array}{l}\text { Réf. } \\
0,1047 * *\end{array}$ & $\begin{array}{l}\text { Réf. } \\
0,1020 * *\end{array}$ \\
\hline $\begin{array}{l}\text { Age : } \\
20-25 \text { ans } \\
25-30 \text { ans } \\
30-35 \text { ans } \\
35-40 \text { ans } \\
40-45 \text { ans } \\
45-50 \text { ans } \\
50-55 \text { ans } \\
55-60 \text { ans } \\
60 \text { ans et plus }\end{array}$ & $\begin{array}{l}\quad \text { Réf. } \\
0,1812 \\
0,4626 \\
0,6921 * * \\
0,5347 \\
0,8116 * * \\
0,5446 \\
0,6528 * \\
0,8082 * *\end{array}$ & $\begin{array}{l}\text { Réf. } \\
0,0274 \\
0,1082 \\
0,2511 \\
0,1749 \\
0,3136 * \\
0,2480 \\
0,2218 \\
0,4063 * *\end{array}$ & $\begin{array}{l}\quad \text { Réf. } \\
0,0149 \\
0,1037 \\
0,2419 \\
0,1753 \\
0,3130 * \\
0,2298 \\
0,2281 \\
0,4100^{* *}\end{array}$ & $\begin{array}{l}\text { Réf. } \\
0,0212 \\
0,1059 \\
0,2465 \\
0,1751 \\
0,3133^{*} \\
0,2389 \\
0,2250 \\
0,4082 * *\end{array}$ \\
\hline $\begin{array}{l}\text { Emploi : } \\
\text { Vacataire } \\
\text { Titulaire } \\
\text { Autres }\end{array}$ & $\begin{array}{l}\text { Réf. } \\
0,6177^{* *} \\
0,4317^{* * *}\end{array}$ & $\begin{array}{l}\text { Réf. } \\
0,1914 * \\
0,2312 * * *\end{array}$ & $\begin{array}{l}\text { Réf. } \\
0,1987 * \\
0,2469 * * *\end{array}$ & $\begin{array}{l}\text { Réf. } \\
0,1950^{*} \\
0,2391^{* * *}\end{array}$ \\
\hline $\begin{array}{l}\begin{array}{l}\text { Enseignement à } \\
\text { distance }\end{array} \\
0 \% \\
0-50 \% \\
50 \% \\
50-100 \% \\
100 \%\end{array}$ & $\begin{array}{c}\text { Réf. } \\
0,8750 * * * \\
1,4841 * * * \\
1,4497 * * * \\
1,9877 * * *\end{array}$ & $\begin{array}{c}\text { Réf. } \\
0,3968 * * * \\
0,7297 * * * \\
1,1652 * * * \\
1,3058 * * *\end{array}$ & $\begin{array}{c}\text { Réf. } \\
0,3749 * * * \\
0,7276 * * * \\
1,2857 * * * \\
1,2456 * *\end{array}$ & $\begin{array}{c}\text { Réf. } \\
0,3858 * * * \\
0,7286 * * * \\
1,2254 * * * \\
1,2757 * * *\end{array}$ \\
\hline $\begin{array}{l}\text { Grade de } \\
\text { recherche : } \\
\text { Non chercheur } \\
\text { Junior } \\
\text { Jeune } \\
\text { Senior } \\
\end{array}$ & \begin{tabular}{l}
\multicolumn{1}{c}{ Réf. } \\
$-0,1557$ \\
$0,2775^{* * *}$ \\
0,1652
\end{tabular} & $\begin{array}{c}\text { Réf. } \\
-0,0846 \\
0,0725 \\
0,0151 \\
\end{array}$ & $\begin{array}{l}\text { Réf. } \\
-0,1772 \\
0,0707 \\
-0,0916 \\
\end{array}$ & $\begin{array}{l}\text { Réf. } \\
-0,0881 \\
0,0716 * \\
0,0097 \\
\end{array}$ \\
\hline
\end{tabular}




\begin{tabular}{|c|c|c|c|c|}
\hline Expert & 0,0802 & $-0,1599$ & 0,0043 & $-0,1685$ \\
\hline \multicolumn{5}{|c|}{ Environnement du travail } \\
\hline $\begin{array}{l}\text { Ordinateur au } \\
\text { travail : } \\
\text { Non } \\
\text { Oui }\end{array}$ & $\begin{array}{c}\text { Réf. } \\
0,1047\end{array}$ & $\begin{array}{c}\text { Réf. } \\
-0,1038\end{array}$ & $\begin{array}{c}\text { Réf. } \\
-0,1080\end{array}$ & $\begin{array}{c}\text { Réf. } \\
-0,1059\end{array}$ \\
\hline $\begin{array}{l}\text { Ordinateur à } \\
\text { domicile : } \\
\text { Non } \\
\text { Oui }\end{array}$ & $\begin{array}{c}\text { Réf. } \\
-0,0727\end{array}$ & $\begin{array}{l}\text { Réf. } \\
-0,2636^{* * *}\end{array}$ & $\begin{array}{c}\text { Réf. } \\
-0,2654 * *\end{array}$ & $\begin{array}{c}\text { Réf. } \\
-0,2645 * *\end{array}$ \\
\hline $\begin{array}{l}\text { Internet en cours: } \\
\text { Non } \\
\text { Oui }\end{array}$ & $\begin{array}{c}\text { Réf. } \\
0,6406 * * *\end{array}$ & $\begin{array}{c}\text { Réf. } \\
0,3083 * * *\end{array}$ & $\begin{array}{c}\text { Réf. } \\
0,3089^{* * *}\end{array}$ & $\begin{array}{c}\text { Réf. } \\
0,3086 * * *\end{array}$ \\
\hline $\begin{array}{l}\text { Equipement } \\
\text { informatique en } \\
\text { salle de cours : } \\
\text { Non } \\
\text { Oui }\end{array}$ & $\begin{array}{c}\text { Réf. } \\
0,6031 * * *\end{array}$ & $\begin{array}{l}\text { Réf. } \\
0,1820 * * *\end{array}$ & $\begin{array}{c}\text { Réf. } \\
0,1753 * * *\end{array}$ & $\begin{array}{c}\text { Réf. } \\
0,1786 * * *\end{array}$ \\
\hline $\begin{array}{l}\text { Nombre } \\
\text { d'étudiants (en } \\
\text { cours) }\end{array}$ & 0,0001 & 0,0001 & $-0,0001$ & 0,0001 \\
\hline $\begin{array}{l}\text { R-squared } \\
\text { Prob }>\text { F }\end{array}$ & $\begin{array}{c}-766,0419 \\
231,14\end{array}$ & $\begin{array}{l}0,3407 \\
0,0000\end{array}$ & $\begin{array}{l}0,3288 \\
0,0000\end{array}$ & $\begin{array}{l}0,3354 \\
0,0000\end{array}$ \\
\hline
\end{tabular}

Réf. : variable de référence

* coefficient significatif au seuil de 10\%, ** coefficient significatif au seuil de $5 \%, * * *$ coefficient significatif au seuil de $1 \%$.

\section{Conclusion générale}

Dans cet article, nous avons cherché à caractériser les usages et les intensités d'usage des principales TIC par les enseignants dans les universités françaises durant l'année universitaire 2005/2006. À partir d'un échantillon biaisé (réponses par Internet), nous avons pu montrer l'existence de nombreuses fractures numériques de premier et de second degré. Nous avons développé un modèle spécifique d'observation de l'intensité d'usage des principales TIC éducatives. 
Relativement à l'adoption des TIC, notre étude tend à montrer une évolution rapide de l'usage des principales technologies génériques (traitement de texte, présentations animées et tableurs) et des limites structurelles à l'adoption de technologies sophistiquées telles que les logiciels de bases de données. Le déplacement de l'intérêt de la recherche vers la variété et l'intensité des usages a permis de montrer l'existence de fractures entre les genres. En moyenne, l'intensité d'usage des TIC des hommes est supérieure de $33 \%$ à celle des femmes. L'ancienneté semble exercer un effet positif sur l'usage et ceci s'explique en grande partie par les effets de réseau et les effets d'apprentissage. L'effet de l'activité de recherche est plus ambigu. L'activité de recherche exerce un effet négatif sur les jeunes enseignants en début de carrière. Cet effet s'inverse et devient positif dès lors que l'on observe les chercheurs confirmés. La titularisation des enseignants exerce également un effet positif sur l'intensité d'usage. La théorie du capital social "universitaire" pourrait être également mobilisée. En effet, l'acquisition de matériel informatique et la disposition de bureau qui leur sont destinés constituent des forces allant vers davantage d'usage des TIC.

Généralement, il convient de mentionner l'absence de dispositifs de formation «ciblés» et généralisés permettant d'accroître l'usage efficace des solutions technologiques. L'absence d'incitation et l'arbitrage concernant le temps est défavorable pour le moment à une telle activité. L'environnement de travail de l'enseignant admet également un impact sur l'usage des TIC. Ainsi, l'équipement des salles en matériel informatique et en Internet agit comme une force allant vers davantage d'usage. Notre simulation n'a pas fourni en revanche d'indications sur l'effet du nombre d'étudiants sur l'intensité d'usage. Les estimations économétriques confirment les principaux résultats obtenus dans l'analyse descriptive. Ce travail nécessite d'être approfondi, au moins, dans trois directions distinctes.

D'une part, une meilleure décomposition des pratiques pédagogiques et une prise en compte des nouvelles solutions apportées par le « rôle participatif » des étudiants conduirait à affiner les résultats et à accentuer les écarts entre groupes d'utilisateurs (enseignants). La prise en compte des interactions entre étudiants et enseignants et leurs influences sur l'intensité d'usage nous semble nécessaire.

D'autre part, nous estimons qu'une analyse plus fine des usages pourrait permettre de le détecter des groupes utilisateurs développant des modèles usages différents. La différence se situerait moins dans l'intensité de l'usage que dans la variété des clusters de technologies adoptées. 
Enfin, la révolution technologique ne pourra pleinement profiter au secteur universitaire sans un changement organisationnel important. L'ampleur de ce changement organisationnel nécessite d'être prise en compte dans la formulation de la future enquête.

\section{REFERENCES}

ALBERO, B. DUMONT, B. (2002) : "Les technologies de l'information et de la communication dans l'enseignement supérieur: pratiques et besoins des enseignants". Rapport ITEM SUP pour le Ministère de la Recherche, Direction de la technologie, Sous-direction des technologies éducatives et des technologiques de l'information et de la communication (SDTETIC), Bureau de l'enseignement supérieur (B3), Paris.

ANDERSSON, S. B. (2006): "Newly qualified teachers' learning related to their use of information and communication technology: a Swedish perspective". British Journal of Educational Technology, 37(5).

BASQUE, J., (2005): "Une réflexion sur les fonctions attribuées aux TIC en enseignement universitaire". Revue internationale des technologies en pédagogie universitaire, 2(1), 30-41.

BECKER, H. J., RAVITZ, J. L., \& WONG, Y. T. (1999): "Teacher and teacherdirected student use of computers and software". (Report No. 3). California, Minnesota: Center for Research on Information Technology and Organizations.

BEN YOUSSEF, A. (2005c): "Information and Communication Technologies and High Education Teachers Training" PSUT-MEDFORIST Conference on e-business and e-learning. May 28-29 Amman, K. of Jordan.

COEN, P. F. (2006): "Construction d'un outil pour évaluer le degré d'intégration des TIC dans l'enseignement". Revue internationale des technologies en pédagogie universitaire, 3(3).

FRAUMANI, B.M.; REINSDROF, M.B.; BROOKS, B.R. (2004): "Price and Real Output Measures for the Education Function of Government: Exploratory Estimates for Primary and Secondary Education", Conference on Research in Income and Wealth, June 29, 2004 Vancouver, BC, Canada. 
GREENE, W.H. (2000): "Econometric Analysis", Fourth Edition, Prentice International Hall Edition.

HAEUW, F. (2002) : “Technologies en formation et compétences des acteurs : adaptation ou transformation?". Éducation permanente, 152, p. 71- 83.

ISSAC, H. (2008): “L’université Numérique”, Rapport Mission Université Numérique.

KARSENTI, T. ; LAROSE, F. (2005) : "L'intégration pédagogique des TIC dans le travail enseignant : recherches et pratiques". Québec, Canada : Presses de l’Université du Québec.

KESSEL, VAN N., et al. (2005): "ICT Education Monitor: Eight years of ICT in schools". The Netherlands, Ministry of Education, Culture and Science.

LAPIERRE, J.; G. GINGRAS, (2001): "Perception des professeurs et des étudiants quant à l'utilisation et l'impact des TIC à l'École polytechnique de Montréal". Département de mathématiques et de génie industriel, École polytechnique de Montréal, $60 \mathrm{p}$.

LAROSE, F. et KARSENTI, T. (2002) : "La place des TIC en formation initiale et continue". Sherbrooke, Canada : Les éditions du Centre de ressources pédagogiques (CRP) de la Faculté d'éducation de l'Université de Sherbrooke.

MADDALA G.; FLORES-LAGAUNES, A. (2001): "Qualitative Response Models", in B. BALTAGI ED., A Companion to Theoretical Econometrics. Oxford: Blackwell.

MEDLIN, B. D. (2001): "The Factors that May Influence a Faculty Member's Decision to Adopt Electronic Technologies in Instruction". Doctoral dissertation, Virginia Polytechnic Institute and State University.

PEA, R. (1985): "Beyond amplification: Using the computer to reorganize mental functioning". Educational Psychologist, 20(4), 167-182.

RABY, C. (2004) : “Analyse du cheminement qui a mené des enseignants du primaire à développer une utilisation exemplaire des Technologies de l'Information et de la Communication (TIC) en classe", Thèse de doctorat en éducation, Université de Québec à Montréal.

ROUX, P.; GALLIANO, D. (2006) : "Les inégalités spatiales dans l'usage des EICT : Le cas des firmes industrielles françaises“, Revue Economique, 57(6), pp. 1449$1475,2006$. 
SHARPE, A. (2004): Ten Productivity Puzzles Facing Researchers, International Productivity Monitor, $\mathrm{Vol}^{\circ} 9$, pp. 15-24.

THOMAS, A. (2000): “Econometric of the qualitative variables”, Dunod, Paris.

UNDERWOOD, J. et al. (2005): "Impact of broadband in schools". UK: Nottingham Trent University, Becta, June 2005. 